\title{
Effective spread and timecourse of neural inactivation caused by lidocaine injection in monkey cerebral cortex
}

\author{
Edward J. Tehovnik *, Marc A. Sommer \\ Department of Brain and Cognitive Sciences, Massachusetts Institute of Technology, E25-634, Cambridge, MA 02139, USA
}

Received 25 June 1996; received in revised form 3 January 1997; accepted 7 January 1997

\begin{abstract}
We studied the effective spread of lidocaine to inactivate neural tissue in the frontal cortex of the rhesus monkey. Injections of $2 \%$ lidocaine at $4 \mu \mathrm{l} / \mathrm{min}$ were made while units were recorded 1 or $2 \mathrm{~mm}$ away. To inactivate units $1 \mathrm{~mm}$ away from the injection site $100 \%$ of the time, $7 \mu \mathrm{l}$ of lidocaine had to be injected. To inactivate units $2 \mathrm{~mm}$ away from the injection site $100 \%$ of the time, $30 \mu 1$ of lidocaine were required. Units were maximally inactivated around $8 \mathrm{~min}$ after the start of a lidocaine injection, and they gradually recovered, regaining most of their initial activity by around $30 \mathrm{~min}$ after the start of an injection. The volume of lidocaine required to inactivate neurons $\geq 90 \%$ of the time could be estimated by the spherical volume equation, $\mathrm{V}=4 / 3 \mathrm{pi}(\mathrm{r})^{3}$. To prolong the inactivation, a slower infusion of lidocaine subsequent to an initial bolus was effective. Saline control injections had no effect. These results allow both a prediction of the timecourse of neural inactivation and an estimate of the spread of neural inactivation following injection of lidocaine into the monkey cerebral cortex. (C) 1997 Elsevier Science B.V.
\end{abstract}

Keywords: Lidocaine; Intracortical injections; Unit recording; Dorsomedial frontal cortex; Rhesus monkey

\section{Introduction}

Neuronal blockade with lidocaine has been used to study a variety of behaviours such as eye movements (Demer and Robinson, 1982; Hikosaka and Wurtz, 1986; Lee et al., 1988; May et al., 1988), drinking (Manani et al., 1995), and the avoidance of noxious stimuli (Urban and Smith, 1994). Lidocaine inactivates neurons by blocking sodium channels (Hille, 1966, 1977; Ritchie, 1979). A major advantage of using this agent over the more conventional permanent lesion technique is that aside from being reversible, this technique does not have problems with compensatory neuronal effects that arise during many weeks of post-lesion recovery (Hikosaka and Wurtz, 1986).

The effective spread of lidocaine and its timecourse of effect has not been studied systematically for differ-

\footnotetext{
* Corresponding author. Tel.: + 1617 2535792; fax: + 1617 2538943; e-mail: tehovnik@wccf.mit.edu
}

ent volumes in the cerebral cortex. We injected different volumes of lidocaine into the dorsomedial frontal cortex of an awake monkey while recording from neurons at different distances away from the injection site. The effective spread of lidocaine conformed to the cubic relationship, volume $=4 / 3$ pi (radius) ${ }^{3}$.

\section{Materials and methods}

\subsection{Subject}

One adult rhesus monkey (Macaca mulatta) was used. Throughout this study food and water were freely available. After testing each day, the animal was returned to the vivarium. The monkey was provided for in accordance with the National Institutes of Health Guide for the Care and Use of Laboratory Animals and the guidelines of the Massachusetts Institute of Technology Committee on Animal Care. 


\subsection{Surgery}

The monkey was anesthetized first with ketamine (10 $\mathrm{mg} / \mathrm{kg})$ and then with pentobarbital $(30 \mathrm{mg} / \mathrm{kg})$ in preparation for sterile surgery. During surgery pentobarbital was administered intravenously. A stainlesssteel post to restrain the head during testing was attached to the skull with head bolts and acrylic cement. A recording chamber was centered and implanted at $25 \mathrm{~mm}$ anterior to the interaural line and $4.0 \mathrm{~mm}$ left of the cerebral midline. The chamber position was biased toward the left dorsomedial frontal cortex, but both hemispheres could be accessed (Fig. 1). After surgery, the monkey was placed on a regimen of antibiotics and pain killer (buprenorphine, $1 \mathrm{mg} / \mathrm{ml} /$ day) for several days.

\subsection{Apparatus for infusion}

Glass-coated platinum-iridium electrodes with an impedance of $0.5-3.0$ megohms at $1 \mathrm{kHz}$ were constructed. Impedance was measured with a $1 \mathrm{kHz}$, nanoampere tester (Bak Electronics, Inc., model A-1B), that was calibrated with a 1.0 megohm resistor. Action potentials were amplified (Bak A-1B) and filtered (Krohn-Hite 3750), displayed on a Tektronix Oscilloscope (model $5103 \mathrm{~N})$, and isolated with a window discriminator (Model 121 Window Discriminator, W.P. Instruments Inc).

The apparatus used to infuse lidocaine (lidocaine hydrochloride, 2\% solution; Steris Laboratories, Inc.,

Fig. 1. A top view of the cortical region injected with lidocaine or saline is shown. The dashed circle circumscribes the region of the dorsomedial frontal cortex (DMFC) over which the recording chamber was situated. The line cutting the circle represents the cerebral midline. The black dots indicate the approximate locations of the injection sites. Many of the sites were injected repeatedly on different test days. The arcuate sulcus (As) and principal sulcus (Ps) are shown along with a scale bar. The top of the figure represents anterior cortex and the bottom of the figure represents posterior cortex.

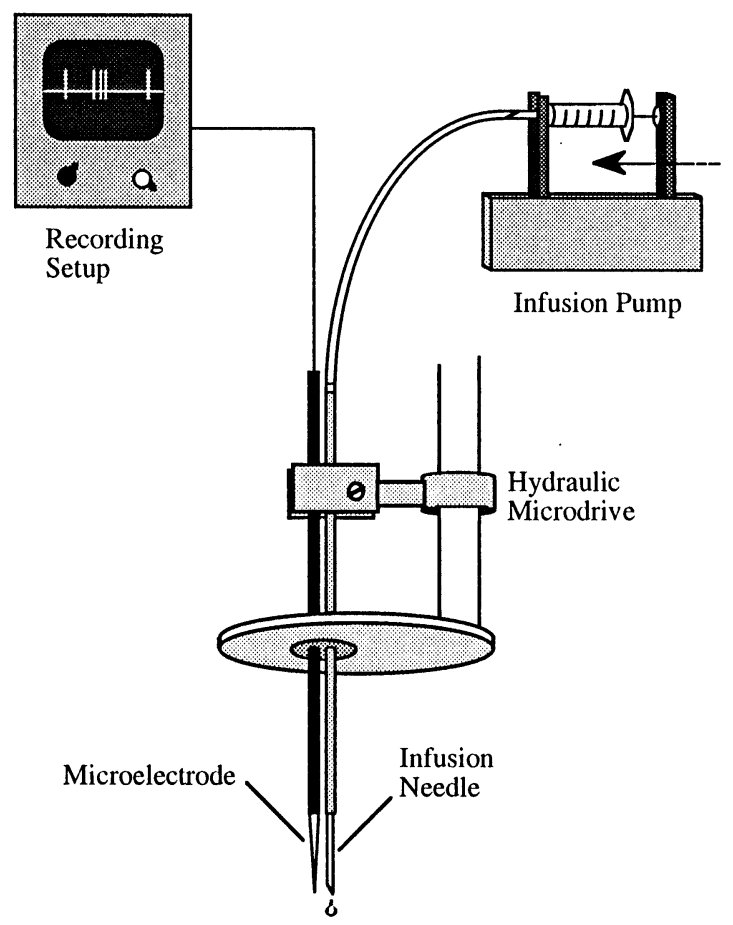

Fig. 2. Schematic of the apparatus used to deliver the lidocaine or saline into the cortex is shown. A hydraulic-microdrive assembly held a cannula and microelectrode in parallel and pushed both through the dura into the brain. Unit activity was monitored with a microelectrode, and lidocaine or saline was infused through the needle tip of the cannula. See Materials and methods (Section 2) for other details.

Phoenix, Arizona, USA) or saline $(0.9 \% \mathrm{Nacl})$ into the cortex is shown (Fig. 2). Using a hydraulic-microdrive assembly, a cannula filled with a pharmacological agent accompanied by a microelectrode was pushed through the dura into the cortex. The cannula was made from a 30-gauge, 16-mm long needle that was epoxied to a 28-gauge stainless-steel tube. Polyethylene tubing (PE50, I.D.: $0.58 \mathrm{~mm}$; O.D.: $0.965 \mathrm{~mm}$ ) was fitted to the end of the 28-gauge stainless-steel tube. About $80 \mathrm{~cm}$ of tubing was connected to a $100 \mu 1$ hamilton syringe, fixed to an infusion pump (Harvard Apparatus Compact Infusion Pump, \# 31537).

The hypodermic syringe and needle, the polyethylene tubing, and the injection cannula were filled with distilled water. The hypodermic syringe was drawn so that $3 \mu 1$ of air were introduced into the cannula tip. The cannula was then filled with the pharmacological agent by drawing $\approx 50 \mu 1$ of agent into it. The $3-\mu 1$ airpocket between the distilled water and agent could be seen as a bubble in the polyethylene tubing. The position of this bubble and the measurement scale (in $\mu 1$ ) on the hypodermic syringe were used to monitor the amount of agent in the injection line.

The filled cannula and a microelectrode were attached to the hydraulic- microdrive assembly. The tip 
of the electrode was aligned with the tip of the cannula; the tips were separated by 1 or $2 \mathrm{~mm}$. The assembly was attached to the recording chamber which was filled with mineral oil. Care was taken to make sure that the bubble in the polyethylene tubing (marking the separation between the distilled water and agent) remained at the same position before and after the assembly was mounted onto the recording chamber. This assured that the mineral oil within the chamber did not enter the cannula due to the volumetric pressure exerted by the oil within the enclosed recording chamber.

The microdrive was now advanced until the recording electrode came in contact with the dura. The electrode and cannula were then pushed through the dura in $5-\mu \mathrm{m}$ steps until units were detected. The electrode and cannula were allowed to settle for $30 \mathrm{~min}$ before descending further to locate units for testing.

\subsection{Parametric testing}

The relationship between the volume of lidocaine infused and the spread and timecourse of neural inactivation was determined. Throughout testing, the monkey, with head fixed, sat in a small illuminated room that was isolated from the infusion pump and from the equipment for unit recording. The monkey performed no task. To reduce the chances of neuronal damage no more than one injection of lidocaine or saline was done within a given test day. Fifty-two separate infusions of lidocaine and 24 separate infusions of saline were done into the dorsomedial frontal cortex. The entire experiment was conducted over a 6 -month period. Once a group of units was encountered that exhibited a steady firing rate, the window discriminator was used to demarcate a lower and upper limit of voltage within which a unit's peak spike amplitude had to occur to trigger an output pulse to a PDP 11/73 computer. The lower limit of voltage was always set above the peak-topeak noise in the baseline recording signal, and the upper limit was set to include several units. Including several units contributed to the stability of the neural firing frequency over time. Large-amplitude units were excluded from the voltage window since they tended to be unstable, often expiring. The rate (in spikes/s) of unit firing was computed every minute (total number of spikes per $\min / 60 \mathrm{~s}$ per $\mathrm{min}$ ) by the PDP computer. The infusion rate of lidocaine or saline, which was controlled by the infusion pump, was held constant at 4 $\mu 1 /$ min unless otherwise specified.

Since the firing frequency of the units at different injection sites varied, the unit activity was normalized by dividing the firing frequency at a given minute following an injection by the baseline firing frequency (Fig. 3A,B). A value less than '1' indicated that the unit activity decreased relative to baseline and a value greater than ' 1 ' indicated that the unit activity increased relative to baseline (Fig. 3B). Neural shutdown was defined as a drop in unit firing frequency to $20 \%$ or lower of baseline firing following an injection (i.e. a drop to 0.2 or lower on the normalized curve, Fig. 3B).

The baseline firing rate had to be stable over many minutes before an injection was done. Since it was necessary to search for units exhibiting stable firing, the injections were often conducted at different depths below the first unit encountered on a pass. Some sites were injected more than once but each injection was always done on a different day, and such a site was never injected more than once a week.

\subsection{Histology}

The location of the cannula/electrode penetrations was determined by noting the location of the well relative to the arcuate sulcus and cortical midline during surgery, and by noting the electrode coordinates on

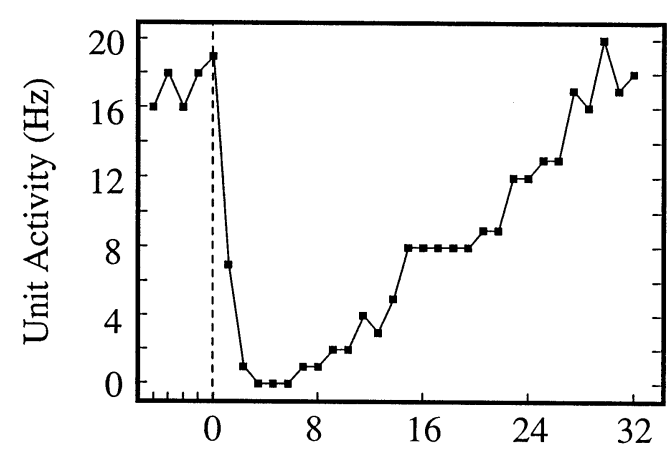

A

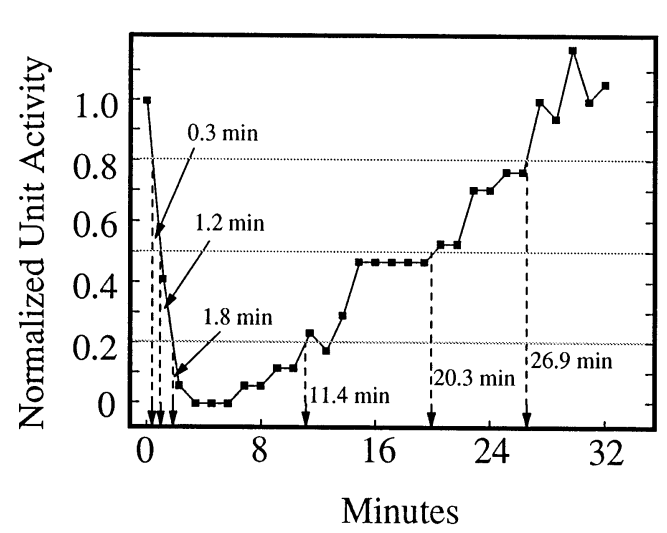

B

Fig. 3. (A) Unit activity is plotted as a function of time following an injection of lidocaine. The dashed vertical line indicates the onset of a $7-\mu 1$ injection of lidocaine. The distance between the cannula tip and electrode tip was $1 \mathrm{~mm}$. (B) The data in (A) are normalized by dividing the firing frequency of the units for a 1-min epoch by the baseline firing frequency immediately before the onset of the injection. The $0.3,1.2$, and $1.8 \mathrm{~min}$ indicated on the declining part of the curve represent the time following the injection that the unit activity decreased to 80,50 , and $20 \%$ of baseline, respectively; the $11.4,20.3$, and 26.9 min indicated on the rising part of the curve represent the time that the unit activity increased to 20,50 , and $80 \%$ of baseline, respectively. 

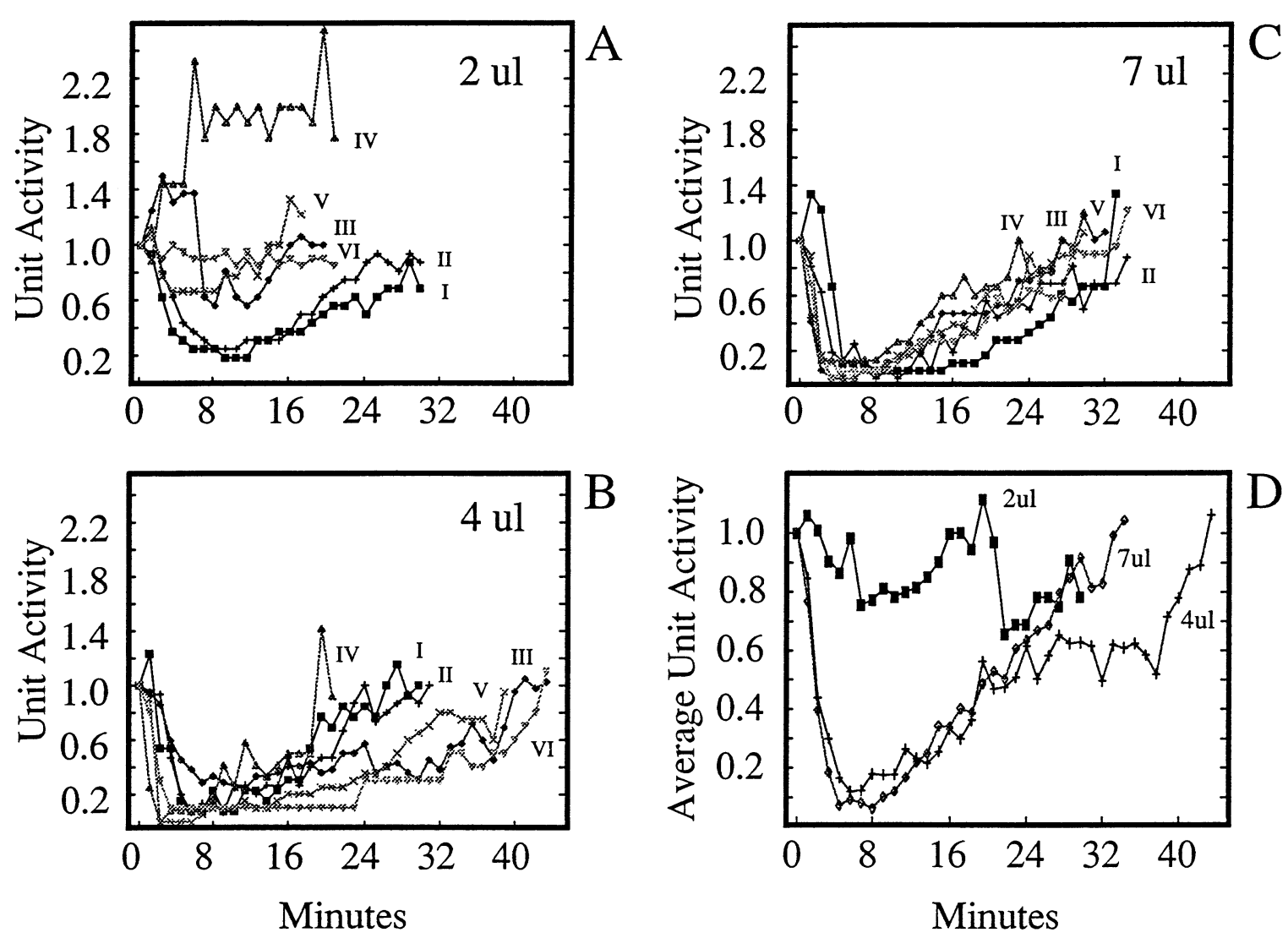

Fig. 4. Normalized unit activity is plotted against time following an injection of lidocaine for six separate injections (I to Vl) using (A) $2 \mu 1$, (B) $4 \mu \mathrm{l}$, and (C) $7 \mu$ l. (D) Average normalized unit activity is shown for the three volumes. The cannula-electrode distance was 1 mm.

a $1 \times 1 \mathrm{~mm}$ grid. After all testing was completed, the monkey was overdosed with pentobarbital, perfused with $0.9 \% \mathrm{NaCl}$, and fixed with $4 \%$ paraformaldehyde. Guide pins were inserted into the cortex at specific positions around the recording chamber. The location of the electrode/cannula sites were estimated relative to the pins. The monkey used in this study had been used in Tehovnik et al. (1994) as monkey A.

\section{Results}

\subsection{Effective spread}

The injection sites are depicted in Fig. 1. The injection depths that were used, at which neurons had acceptably stable and vigorous firing rates, ranged from 0.5 to $1.5 \mathrm{~mm}$ below the first unit encountered on a descent. Baseline firing frequencies ranged from 10 to $50 \mathrm{~Hz}$. Within minutes of the start of a lidocaine infusion, the neural firing rate dropped rapidly (Fig. 3A,B). After that, the neural activity gradually recovered. Although eight or so injections of agent were made at the same cortical entrance point, with repeated injections there was no obvious reduction in unit activity at the sites below an entrance point.
Normalized unit activity is plotted as a function of time after a lidocaine injection for different conditions (Figs. 4 and 5). Fig. 4A-C illustrate the effect of injecting 2, 4, and $7 \mu 1$ of lidocaine when the cannula and electrode tips were spaced by $1 \mathrm{~mm}$. For each volume, six separate injections (I to Vl) were performed. To achieve shutdown of the neurons (a drop in unit activity to $20 \%$ or less of baseline firing: 0.2 or less on a normalized graph) in 6 of 6 cases, $7 \mu 1$ of lidocaine were necessary. The average drop in activity for the 2 , 4 , and $7 \mu 1$ of lidocaine is shown in Fig. 4D, with $2 \mu 1$ having a negligible effect on the cells and 4 and $7 \mu 1$ having a large effect. Eight min following an injection of lidocaine, the neural activity did not exhibit a significant drop from baseline when $2 \mu 1$ was injected $(t(5)=$ $0.9, p>0.05)$, but did exhibit a significant drop when 4 or $7 \mu 1$ was injected $(t(5)=23.6, p<0.05 ; t(5)=36.7$, $p<0.05)$.

Fig. 5A-E show the effect of injecting 4, 7, 10, 20, and $30 \mu 1$ of lidocaine when the cannula and electrode tips were spaced by $2 \mathrm{~mm}$. To get shutdown in 6 of 6 cases, $30 \mu 1$ of lidocaine were required. The average drop in unit activity for $4,7,10,20$, and $30 \mu 1$ of lidocaine is shown (Fig. 5F), with the magnitude of the drop increasing systematically with volume. Eight min- 

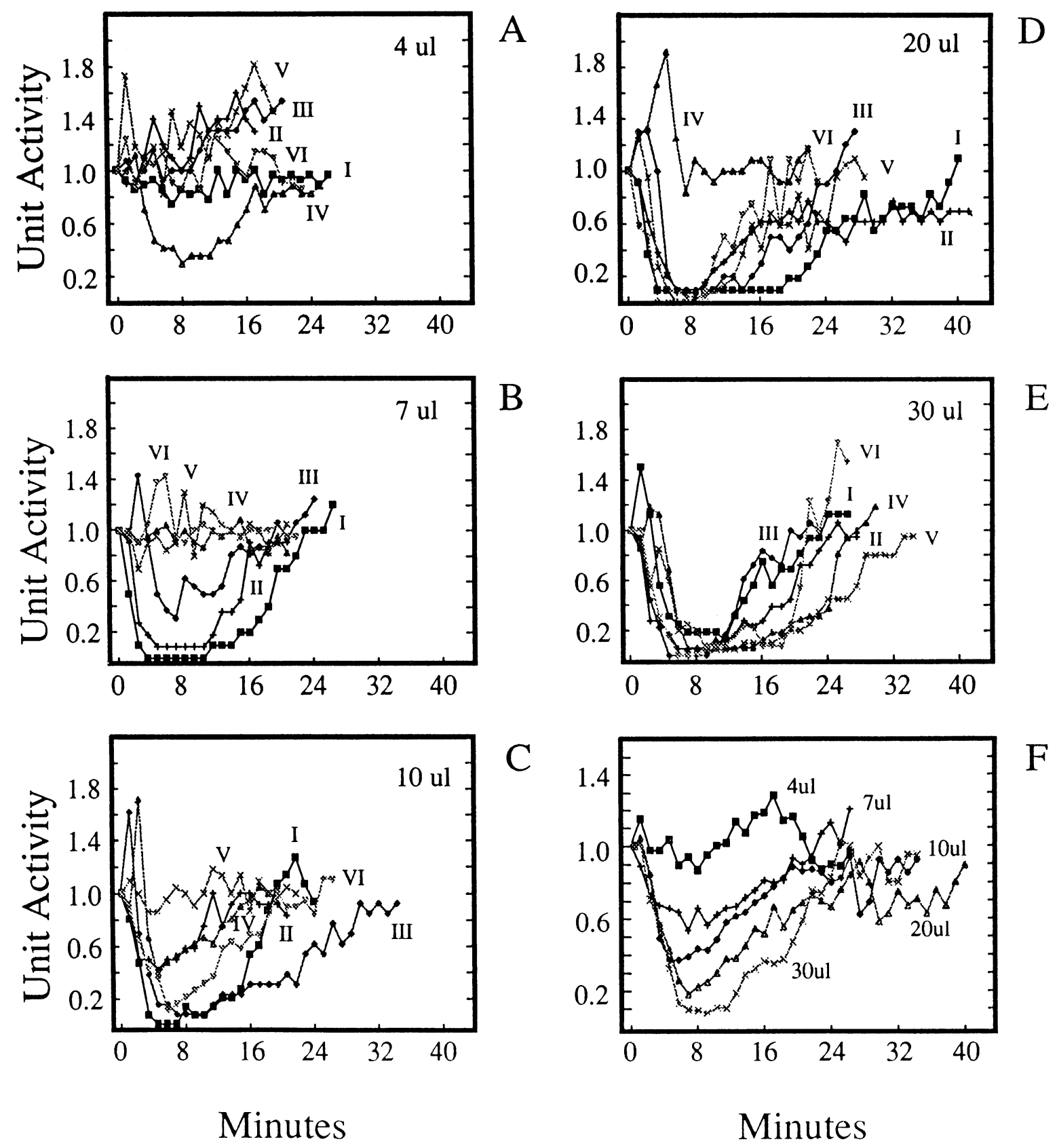

Fig. 5. Normalized unit activity is plotted against time following an injection of lidocaine for six separate injections (I to VI) using (A) $4 \mu 1$, (B) $7 \mu 1$, (C) $10 \mu 1$, (D) $20 \mu 1$, and (E) $30 \mu$ l. (F) Average normalized unit activity is shown for the five volumes. The cannula-electrode distance was $2 \mathrm{~mm}$.

utes following an injection of lidocaine, the neural activity did not exhibit a significant drop from baseline when 4 or $7 \mu 1$ was injected $(t(5)=1.1, p>0.05 ; t(5)=$ $2.2, p>0.05)$, but did exhibit a significant drop when 10 , 20, or $30 \mu 1$ was injected $(t(5)=6.1, p<0.05$; $t(5)=5.1, p<0.05 ; t(5)=24.5, p<0.05)$.

The effect of the saline injections on the average unit activity is illustrated in Fig. 6. When the cannula and electrode tips were spaced by $1 \mathrm{~mm}, 2,4$, and $7 \mu 1$ of saline had a negligible effect on the unit activity. Likewise, when the cannula and electrode tips were spaced by $2 \mathrm{~mm}, 4,7,10$, and $20 \mu 1$ of saline had a negligible effect, although the variability was greater. The $30 \mu 1$ injection caused a drop in unit activity, but the drop was well above the 0.2 level on the normalized graph, the criterion used for shutdown. Eight min following an 
injection of saline, the neural activity exhibited a significant drop from baseline only when $30 \mu 1$ was injected $(t(2)=8.0, p<0.5)$, but not when lesser volumes were injected.

\subsection{Temporal properties}

For each case where there was shutdown following a lidocaine injection, the time for a decline in unit activity to 80,50 , and $20 \%$ of baseline firing following an injection is shown in Fig. 7A. The method used to derive the times is illustrated in Fig. 3B. The average time for a drop in unit activity to 80,50 , and $20 \%$ of baseline in cases where the cannula and electrode were spaced by $1 \mathrm{~mm}$ was $1.3,2.2$, and $3.4 \mathrm{~min}$, respectively. The average time for a drop to 80,50 , and $20 \%$ of baseline in cases where the cannula and electrode were spaced by $2 \mathrm{~mm}$ was $1.9,2.9$, and $4.4 \mathrm{~min}$, respectively. Shutdown occurred within $5 \mathrm{~min}$ when lidocaine was injected with a 1 or $2 \mathrm{~mm}$ cannula-electrode separation.

For each case where there was shutdown following a lidocaine injection, the time for recovery in unit activity to 20,50 , and $80 \%$ of baseline following an injection is shown in Fig. 7B. The method used to derive the times is illustrated in Fig. 3B. The average time for an increase in unit activity to 20,50 , and $80 \%$ of baseline

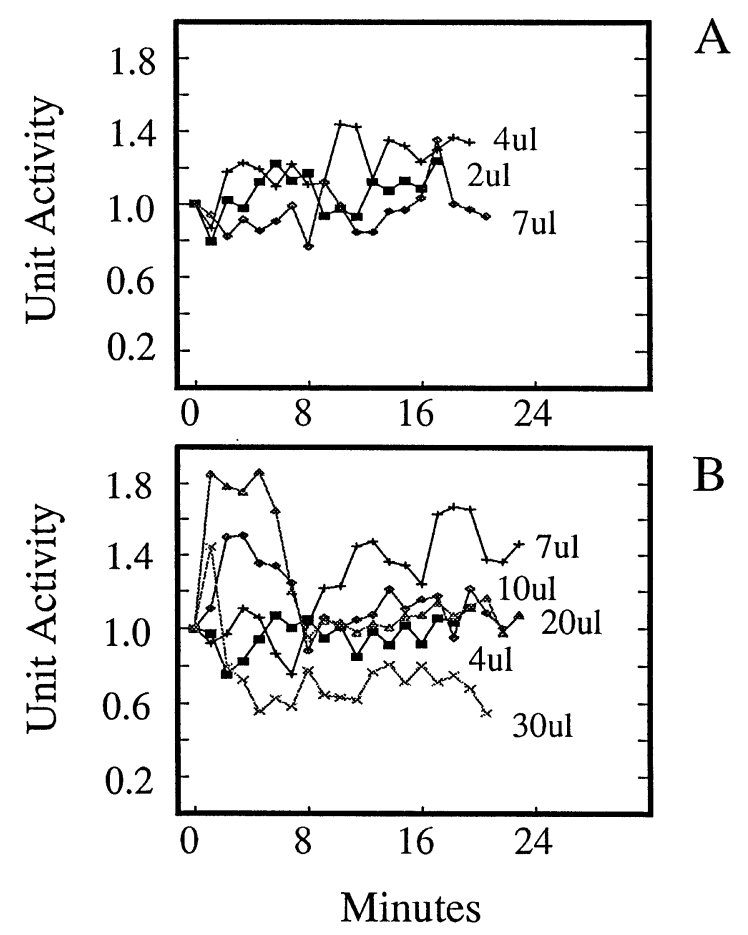

Fig. 6. Average normalized unit activity is plotted against time following an injection of saline. Each curve represents an average of three separate injections. (A) The electrode-cannula distance was 1 mm using 2, 4, and $7 \mu \mathrm{l}$ of saline. (B) The electrode-cannula distance was $2 \mathrm{~mm}$ using $4,7,10,20$, and $30 \mu \mathrm{l}$ of saline.
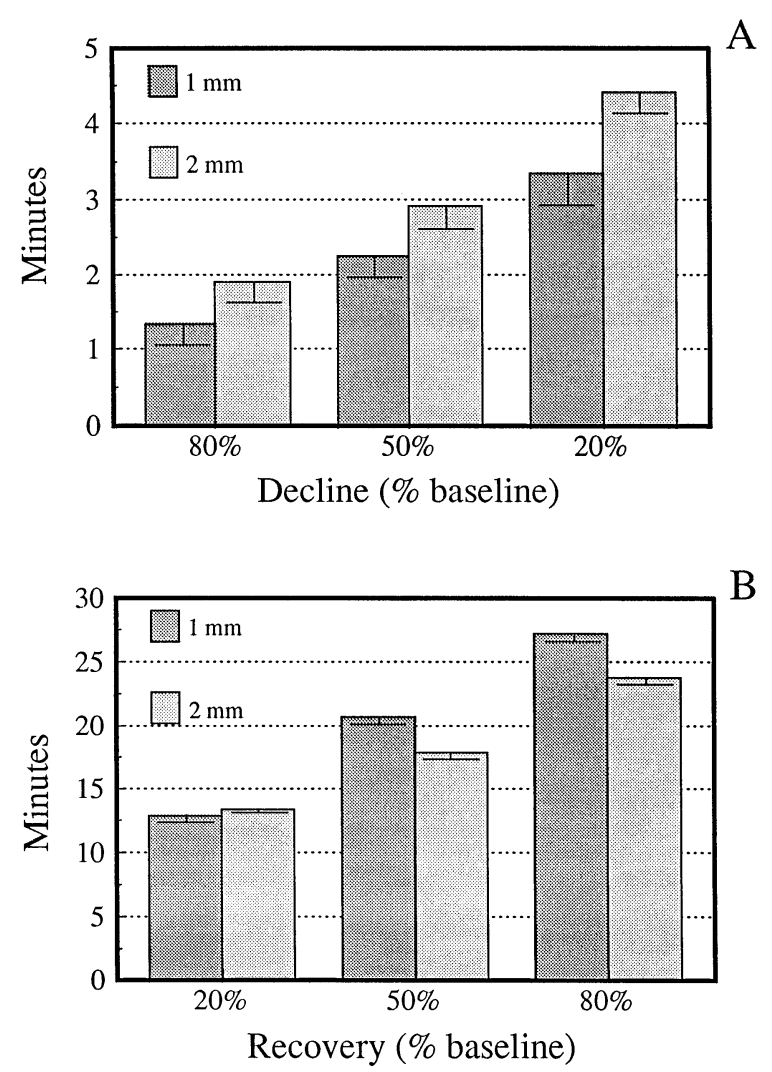

Fig. 7. (A) The average time for a decline in unit activity to 80,50 , and $20 \%$ of baseline is shown for injections of lidocaine for a 1-and 2-mm cannula-electrode separation. (B) The average time for a recovery in unit activity to 20,50 , and $80 \%$ of baseline is shown for injections of lidocaine for a 1- and 2-mm cannula-electrode separation. The averages are based on data in Fig. 4a-c and Fig. 5a-e). Data from Fig. 5a were not used since none of the curves declined to $20 \%$ of baseline. Standard errors are shown for each bar graph. A $t$-test revealed no significant differences $(p>0.05)$ between the 1 and $2 \mathrm{~mm}$ conditions. See text for other details.

in cases where the cannula and electrode were spaced by $1 \mathrm{~mm}$ was $12.9,20.7,27.2 \mathrm{~min}$, respectively. The average time for an increase to 20,50 , and $80 \%$ of baseline in cases where the cannula and electrode were spaced by $2 \mathrm{~mm}$ was 13.3, 17.9, $23.8 \mathrm{~min}$, respectively. Recovery to $80 \%$ of baseline occurred within $30 \mathrm{~min}$ when lidocaine was injected with a 1 or $2 \mathrm{~mm}$ cannulaelectrode separation.

\subsection{Probability of inactivation}

The probability of neuronal shutdown to $20 \%$ of baseline varied as a function of the volume of lidocaine injected and it varied as a function of the distance between the cannula and electrode (Fig. 8A). Three $\mu 1$ of lidocaine were required for a $50 \%$ probability of shutdown when the cannula and electrode were spaced by $1 \mathrm{~mm}$, and $10 \mu 1$ were required for a $50 \%$ probability of shutdown when the cannula and electrode were spaced by $2 \mathrm{~mm}$. The volumes of lidocaine required to 
achieve a $50,70,90$, and $100 \%$ probability of shutdown at 1 and $2 \mathrm{~mm}$ from the cannula are illustrated (Fig. $8 b)$. Notice that the curve representing the volume of a sphere overlaps with the 90 and $100 \%$ probability lines for shutdown using lidocaine, suggesting that this relationship can be used to estimate the effective spread of lidocaine within cortex.

\subsection{Continuous infusion}

In the experiments described thus far, lidocaine was injected into the cortex at an infusion rate of $4 \mu 1 / \mathrm{min}$ for less than $8 \mathrm{~min}$. The cells tended to begin their recovery as early as 12 to $14 \mathrm{~min}$ following an injection (e.g. Fig. 7B at $20 \%$ of baseline). We next tested to see if we could extend the period of neuronal shutdown by imposing a slow drip of lidocaine after the normal 4 $\mu \mathrm{l} / \mathrm{min}$ dose was finished. Eight $\mu 1$ of lidocaine was injected at $4 \mu \mathrm{l} / \mathrm{min}$ followed by lidocaine delivery at $0.1,0.2$, and $0.3 \mu 1 / \mathrm{min}$ for $15 \mathrm{~min}$ (Fig. 9A). The time

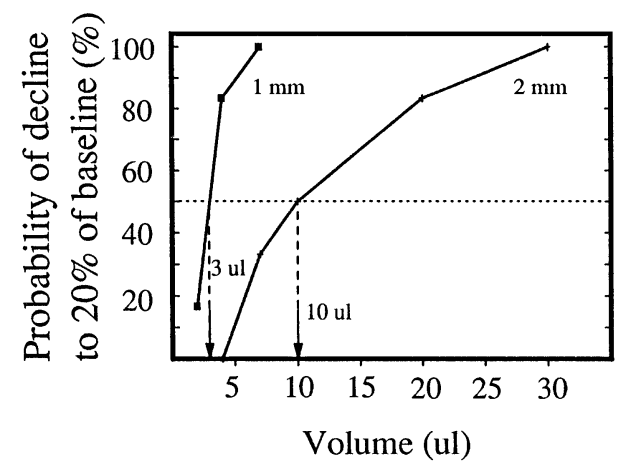

A

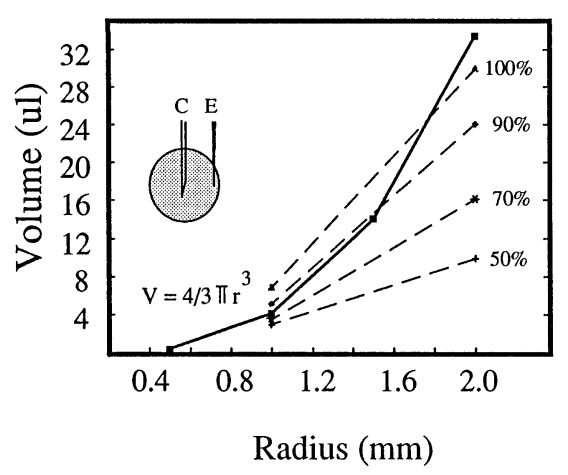

$\mathrm{B}$

Fig. 8. (A) The probability of neuronal shutdown to $20 \%$ of baseline is plotted as a function of the volume of lidocaine injected for cannula-electrode distances of 1 and $2 \mathrm{~mm}$. Each data point, derived from Fig. 4A-C and Fig. 5A-E, is based on the six separate injections of lidocaine. (B) Volume of lidocaine required to achieve $50,70,90$, and $100 \%$ probability of shutdown (dashed lines) is plotted for electrode-needle tip separation (radius), as estimated from the curves in (A). The volumetric spread of a liquid from a cannula $\mathrm{C}$ to an electrode $\mathrm{E}$ is plotted (black curve) using the relationship, $\mathrm{V}=4$ / 3 pi $(r)^{3}$, where $\mathrm{V}$ is volume and $\mathrm{r}$ is radius.
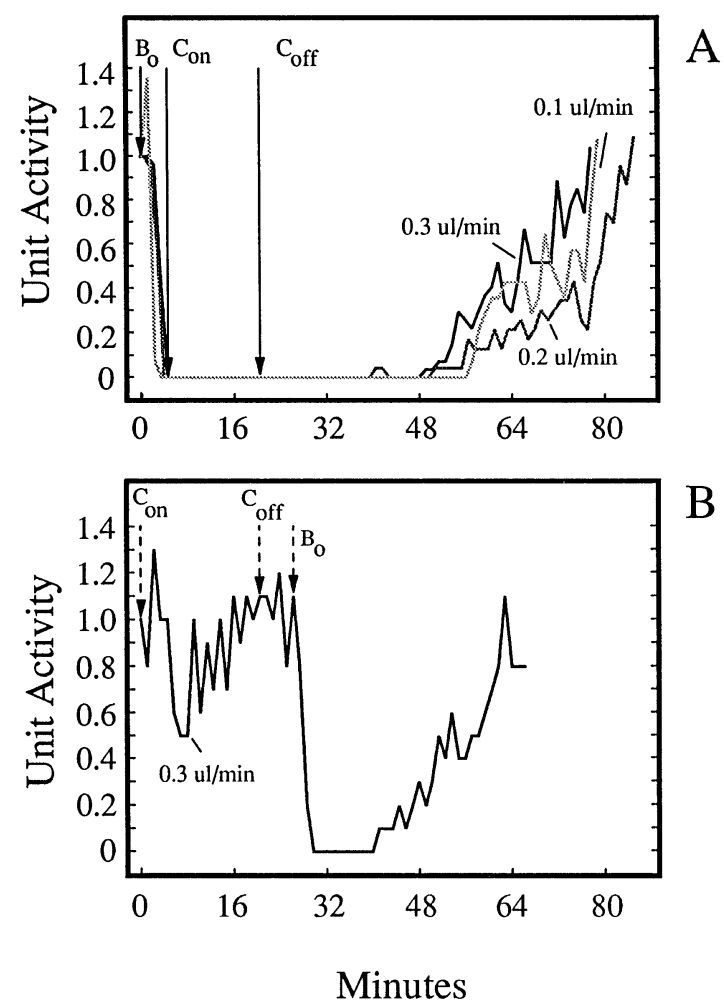

B

Fig. 9. Normalized unit activity is plotted as a function of time following the start of a lidocaine injection. (A) Results from three separate injections are shown. Eight $\mu 1$ of lidocaine were injected at a rate of $4 \mu \mathrm{l} / \mathrm{min}$ at time $\mathrm{B}_{\mathrm{o}}$, and three continuous infusion rates were tested: $0.1,0.2$, and $0.3 \mu 1 / \mathrm{min}$. At time $\mathrm{C}_{\text {on }}$ the continuous drip was started and at time $\mathrm{C}_{\text {off }}$ the continuous drip was stopped. (B) A continuous infusion rate of $0.3 \mu \mathrm{l} / \mathrm{min}$ of lidocaine was delivered before a bolus of $8 \mu 1$ of lidocaine was injected.

of neuronal shutdown lasted between 52 and $62 \mathrm{~min}$ (i.e. the time the curves in Fig. 9A increased to $20 \%$ of baseline). The different drip rates used did not affect this time in any systematic way. When a low rate of lidocaine infusion was administered before the bolus was delivered, there was a slight effect (Fig. 9B). Shutdown to $20 \%$ of baseline, however, was evident only once the normal bolus at $4 \mu \mathrm{l} / \mathrm{min}$ was delivered.

\section{Discussion}

\subsection{Temporal properties}

Neuronal shutdown following an injection of lidocaine into the dorsomedial frontal cortex was immediate, occurring within $5 \mathrm{~min}$ of starting an injection and recovering within $30 \mathrm{~min}$. These finding coincide with previous studies where oculomotor (Demer and Robinson, 1982; May et al., 1988) and evoked potential (McGee et al., 1991) measures were used to assess the onset of an effect of lidocaine and recovery thereof. 
Anywhere from 1 to $30 \mu 1$ of lidocaine were injected in these studies, volumes comparable to those used in the current study. The concentration of lidocaine used in these studies was somewhat higher at $4 \%$ rather than at $2 \%$ as used in the current study. Also most injections were made into subcortical structures. The relationship between the temporal effects of lidocaine and its concentration has not been tested. It is common for investigators to use a 1 to $4 \%$ solution for inactivating neural tissue (e.g. Sandkühler and Gebhart, 1984; May et al., 1988).

The blocking effects of lidocaine are largely over by $30 \mathrm{~min}$. Testing the behavioural effects of neuronal inactivation for 30 or so $\mathrm{min}$ allows one to obtain behavioural measures before, during, and after inactivation on the same day with minimal complications arising from an animal's fatigue (Sommer and Tehovnik, in press). One, however, is restricted temporally when using lidocaine, which is not true when using agents such as muscimol (Hikosaka and Wurtz, 1985). To overcome this problem we have found that once a bolus of lidocaine has been administered, the suppressive effects of lidocaine can be prolonged to over $1 \mathrm{~h}$ by continuously infusing lidocaine into the brain at a low rate (e.g. $<0.3 \mu 1 / \mathrm{min}$ ) for many tens of minutes.

\subsection{Volumetric spread of lidocaine}

The results suggest that the relationship between the volume of lidocaine (in $\mu 1$ or $\mathrm{mm}^{3}$ ) and its effective spread (in $\mathrm{mm}$ ) conforms to the cubic relationship, volume $=4 / 3$ pi (radial spread $)^{3}$. This conclusion is based on volumes from 2 to $30 \mu 1$ tested on neurons 1 and $2 \mathrm{~mm}$ away from a cannula tip.

While measuring the effects of lidocaine on the unit activity within the lateral geniculate nucleus, Malpeli and Schiller (1979) determined the radial spread of 20 and $50 \mathrm{nl}$ of $2 \%$ lidocaine to be 100 and $200 \mu \mathrm{m}$. Using the cubic relationship, 20 and $50 \mathrm{nl}$ of lidocaine should inactivate neurons within 170 and $230 \mu \mathrm{m}$ from the cannula tip. By measuring changes in the current threshold of stimulation-evoked responses before and after injections of 1 or $4 \%$ lidocaine into the medulla or spinal cord, Sandkühler and Gebhart (1984) and Sandkühler et al. (1987) determined that $500 \mathrm{nl}$ of lidocaine spreads 435 or $500 \mu \mathrm{m}$ from the cannula tip and that $1000 \mathrm{nl}$ of lidocaine spreads $750 \mu \mathrm{m}$ from the cannula tip. Using the cubic relationship, $500 \mathrm{nl}$ of lidocaine should inactivate neurons within $490 \mu \mathrm{m}$ from the cannula tip and $1000 \mathrm{nl}$ of lidocaine should inactivate neurons within $620 \mu \mathrm{m}$ from the cannula tip. Accordingly, the cubic relationship not only accounts for the estimates of the current study that uses large volumes of lidocaine (i.e. 2 to $30 \mu 1$ ) but it also accounts for the estimates of other studies that use extremely small volumes (i.e. $<1 \mu 1$ ). Fig. 10 shows that the estimates of the current study along with the estimates of Malpeli and Schiller (1979); Sandkühler and Gebhart (1984), and Sandkühler et al. (1987) correspond well to the cubic relationship. The number of studies estimating the effective spread of lidocaine is small. Future studies will need to be done to further ascertain the strength of the cubic relationship.

\subsection{Damage}

As already discussed, following an injection of lidocaine the effects are largely over by $30 \mathrm{~min}$. Regardless of the volume of the injection up to $30 \mu 1$, complete neuronal recovery was typical, indicating that the neurons at a recording site $2 \mathrm{~mm}$ from the cannula tip were largely unaffected. We did find, however, that under these conditions, saline of $30 \mu 1$, but not 10 or $20 \mu 1$, produced significant neuronal shutdown that remained so for tens of minutes. This suggests that at an infusion rate of $4 \mu 1 / \mathrm{min}$ (which was the standard used in this study) some mechanical damage due to volume displacement might occur. Although we never recorded the neural activity close to the cannula tip, Demer and Robinson (1982) have suggested that damage at the canula tip is unavoidable even at infusion rates of 1 or $2 \mu \mathrm{l} / \mathrm{min}$ using volumes less than $12 \mu 1$. Unlike the study of Demer and Robinson (1982), however, our injections were never made into the same site more than once per day and the time between successive injections into one site was always greater than 1 week.

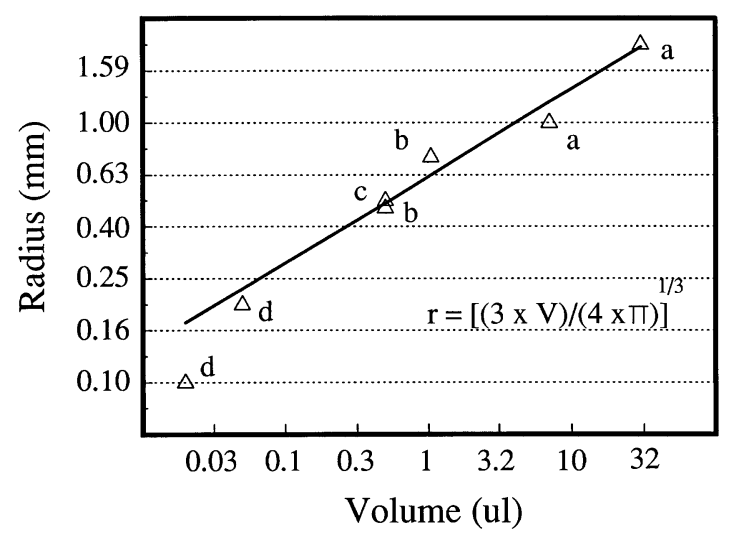

Fig. 10. The effective radial spread of lidocaine is plotted as a function of volume using a log-log plot. The individual data points (open triangles) are from (a) the current study (Fig. 8B: volumes producing $100 \%$ shutdown for cannula-electrode separations of 1 and $2 \mathrm{~mm}$ ), from (b) Sandkühler et al. (1987), and from (c) Sandkühler and Gebhart (1984), and from Malpeli and Schiller (1979). The black line cutting the data points represents the volume of a sphere, $\mathrm{r}=[(3 \times \mathrm{V}) /(4 \times \mathrm{pi})]^{1 / 3}$, where $\mathrm{r}$ is radius and $\mathrm{V}$ is volume. 


\subsection{Radioactive spread of lidocaine}

Martin (1991) injected $1 \mu 1$ of $\left[{ }^{14} \mathrm{C}\right]$ lidocaine $(4 \%)$ into subcortical regions of the rat brain. The radioactive lidocaine was found to spread radially from the cannula tip in coronal brain sections. The radioactive lidocaine was indistinguishable from background beyond $60 \mathrm{~min}$ post-injection. Our findings suggest that lidocaine is ineffective at disrupting neural activity beyond $30 \mathrm{~min}$ post-injection. The maximal radioactive spread of $1 \mu 1$ of $4 \%$ lidocaine was estimated by Martin to be roughly $1.8 \mathrm{~mm}$ from the cannula tip, which was corroborated by a drop in glucose utilization. According to the cubic relationship, to inactivate tissue $1.8 \mathrm{~mm}$ away from the cannula tip, $24 \mu 1$ of lidocaine would be required, and $1 \mu 1$ of lidocaine should inactivate tissue $0.62 \mathrm{~mm}$ away from the cannula tip. Thus, the simple spread of lidocaine molecules is not equivalent to the effective spread of lidocaine as defined with unit recording. This indicates that the concentration of lidocaine molecules necessary for detection of the substance is less than the concentration necessary to effectively inactivate a neuron. We suggest that investigators be careful in using the spread of labelled molecules in interpreting the amount of tissue affected by the pharmacological effect of those molecules.

\subsection{Inactivating cortical tissue in macaque monkeys}

In order to inactivate a 2-mm thick cortex, two conditions are critical. First, the cannula should be positioned in the deep layers of cortex, and second, between 8 and $16 \mu$ l of lidocaine should be injected (Fig. 11). The deep placement assures that the cannula is deep enough so as to avoid superficial injections that might fail to inactivate cortex due to leakage up the cannula shaft. A deep placement can be accomplished by pushing the cannula/electrode assembly between 1 and $2 \mathrm{~mm}$ below the first unit encountered on a pass. Volumes of 8-16 $\mu 1$ make sure that all the cortical layers are being inactivated, especially the output layers. In order to produce reliable deficits in the execution of saccadic eye movements, between 8 and $18 \mu 1$ of lidocaine had to be injected deep within the frontal eye fields of macaque monkeys (unpublished observations, also see Sommer and Tehovnik, in press). Volumes below $4 \mu 1$ were ineffective at producing an oculomotor deficit. It is noteworthy that volumes less than $4 \mu 1$ are sufficient to produce an oculomotor deficit following an injection of lidocaine into subcortical regions such as the superior colliculus (Hikosaka and Wurtz, 1986; Lee et al., 1988). Whether this difference in effective volume is related to a difference in the neuronal volume devoted to oculomotor control within the frontal eye fields and superior colliculus, or whether it is related to less lidocaine leaking up the electrode shaft during deep subcortical injections, is not known.

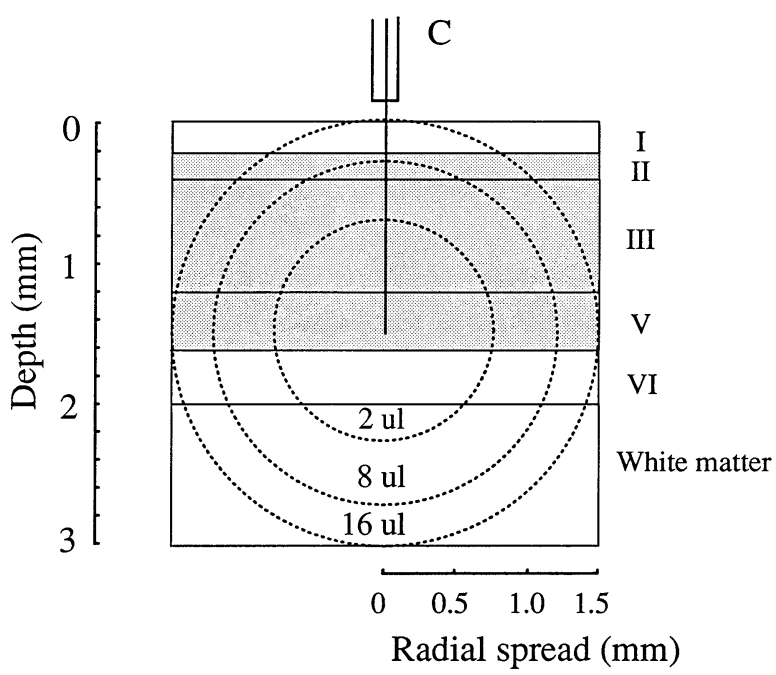

Fig. 11. Illustrated is a 2-mm thick cerebral cortex (layers I to VI), which is the approximate thickness of macaque dorsomedial frontal cortex (Winters et al., 1969). The layers containing cells are indicated in grey. With a cannula tip located at $1.5 \mathrm{~mm}$ below the first unit encountered on a pass, the volume of lidocaine injected to inactivate different portions of cortex is depicted using 2 , 8, and $16 \mu 1$ of lidocaine based on the relationship, volume $=4 / 3$ pi (radius) ${ }^{3}$.

Although lidocaine has advantages such as a quick onset, a short time of affect, and causing a complete deadening of tissue, its major disadvantage is that it inactivates axons along with the cell bodies (Fig. 11). Lidocaine inactivates all axons terminating in or passing through an injected area of cortex. It is imperative, therefore, that lidocaine be used in combination with an agent that disables only cell bodies to verify that the effect of lidocaine inactivation can be attributed to activity of cells residing in the injected area. Muscimol is an agent that affects cell bodies without interfering with the conduction of axons. Muscimol, however, is limited given its long time of effect (Hikosaka and Wurtz, 1985; Sommer and Tehovnik, in press) and unknown effective spread properties.

\subsection{Conclusion}

The blocking effect of lidocaine is largely over after $30 \mathrm{~min}$. The effective spread of lidocaine within neural tissue can be described using the cubic relationship, volume $=4 / 3 \mathrm{pi}(\mathrm{r})^{3}$. This relationship is robust for describing both small- and large-volume injections. By noting the timecourse and effective spread of lidocaine in cortical tissue, investigators will be better able the assess the significance of behavioural deficits, or lack of, arising from targeted injections of lidocaine into different cortical areas. 


\section{Acknowledgements}

This work was supported by NIH EY08502 to P.H. Schiller.

\section{References}

Demer, J.L. and Robinson, D.A. (1982) Effects of reversible lesions and stimulation of olivocerebellar system on vestibulocular reflex plasticity, J. Neurophysiol., 47: 1084-1107.

Hikosaka, O. and Wurtz, R.H. (1985) Modification of saccadic eye movements by GABA-related substances. I. Effect of muscimol and bicuculline in monkey superior colliculus, J. Neurophysiol., 53: $337-352$.

Hikosaka, O. and Wurtz, R.H. (1986) Saccadic eye movements following injection of lidocaine into the superior colliculus, Exp. Brain Res., 61: 531-539.

Hille, B. (1966) Common mode of action of three agents that decrease the transient change in sodium permeability in nerves, Nature, 210: $1220-1222$.

Hille, B. (1977) The pH-dependent rate of action of local anesthetics on the node of Ranvier, J. Gen. Physiol., 69: 475-496.

Lee, C., Rohrer, W.H. and Sparks, D.L. (1988) Population coding of saccadic eye movements by neurons in the superior colliculus, Nature, 332: 357-360.

Malpeli, R.J. and Schiller, P.H. (1979) A method of reversible inactivation of small regions of brain tissue, J. Neurosci. Methods, 1: $143-151$.

Martin, J.H. (1991) Autoradiographic estimation of the extent of reversible inactivation produced by microinjection of lidocaine and muscimol in the rat, Neurosci. Lett., 127: 160-164.
Manani, J.V., Beltz, T.G. and Johnson, A.K. (1995) Effects of lidocaine injections into the lateral parabrachial nucleus on dipsogenic and pressor responses to central angiotension II in rats, Brain Res., 695: 250-252.

May, J.G., Keller, E.L. and Suzuki, D.A. (1988) Smooth-pursuit eye movement deficits with chemical lesions in the dorsolateral pontine nucleus of the monkey, J. Neurophysiol., 59: 952-977.

McGee, T., Kraus, N., Comperatore, C. and Nicol, T. (1991) Subcortical and cortical components of the MLR generating system, Brain Res., 544: 211-220.

Ritchie, J.M. (1979) A pharmacological approach to the structure of sodium channels in myelinated axons, Annu. Rev. Neurosci., 2: $341-362$.

Sandkühler, J. and Gebhart, G.F. (1984) Relative contributions of the nucleus raphe magnus and adjacent medullary reticular formation to the inhibition by stimulation in the periaqueductal gray of a spinal nociceptive reflex in the pentobarbital-anesthetized rat, Brain Res., 305: 77-87.

Sandkühler, J., Maisch, B. and Zimmersmann, M. (1987) The use of local anaesthetic microinjections to identify central pathways: a quantitative evaluation of the time course and extent of the neural block, Exp. Brain Res., 68: 168-178.

Sommer, M.A. and Tehovnik, E.J. Reversible inactivation of macaque frontal eye field. Exp. Brain Res. (in press).

Tehovnik, E.J., Lee, K-M. and Schiller, P.H. (1994) Stimulationevoked saccades from the dorsomedial frontal cortex of the rhesus monkey following lesions of the frontal eye fields and superior colliculus, Exp. Brain Res., 98: 179-190.

Urban, M.O. and Smith, D.J. (1994) Nuclei within the rostral ventromedial medulla mediating morphine antinociception from the periaqueductal gray, Brain Res., 652: 9-16.

Winters, W.D., Kado, R.T. and Adey, W.R. (1969) A Stereotaxic Brain Atlas for Macaca nemestrina, University of California Press, Los Angeles. 